\title{
Increased activity of inflammasomes as osmosensors as cause of cerebral edema in diabetic ketoacidosis
}

\author{
Michael Eisenhut* \\ Luton and Dunstable University Hospital NHS Foundation Trust, Lewsey Road, Luton, LU4ODZ, United Kingdom
}

\begin{abstract}
Diabetic ketoacidosis (DKA) related cerebral edema (CE) is associated with a high mortality and rate of neurodisability and neurocognitive difficulties. In $54 \%$ of children with DKA there is subclinical evidence of CE on MRI studies. It is known that DKA is accompanied by a systemic inflammatory response characterized by elevated cytokine levels including interleukin-1 (IL-1) and interleukin-6.

IL-1 has been linked to the pathogenesis of CE. Inflammasomes like nucleotide binding domain and leucine-rich repeat containing proteins 3 (NRLP3) are multiprotein complexes generating the active form of $\mathrm{IL}-1$ in response to high glucose levels. The resulting hypothesis is that increased activity of inflammasomes as osmosensors is the cause of CE in diabetic ketoacidosis. Confirmation of the hypothesis could be achieved by correlation of IL-1 and NRLP3 levels and expression with degree of subclinical CE on MRI imaging and peripheral blood glial fibrillary acidic protein levels (a marker of brain injury). If confirmed future randomized controlled trials could use NRLP3 inhibitors and IL-1 antagonists as auxiliary treatment in DKA and measure neurocognitive outcome.
\end{abstract}

\section{Background}

Clinically manifest cerebral edema (CE) in diabetic ketoacidosis is rare in high income countries ( 0 to $5.5 \%$ of cases) and significantly more common in low income countries (24 to $26 \%$ of cases) [1]. Mortality of severe diabetic ketoacidosis (DKA) is high with more than $20 \%$ and survivors are at a significant risk of at least 27\% [2] of permanent neurodisability. The pathogenesis of the associated $\mathrm{CE}$ is unclear. There is subclinical and mild, not formally diagnosed CE, which was detected in a previous study, which measured the intercaudate width of the frontal horns of the lateral ventricles using magnetic resonance imaging (MRI) in 41 patients with DKA during treatment and after recovery. The lateral ventricles were significantly smaller during DKA treatment in 22 children (54\%) who were more likely to have mental status abnormalities than those without narrowing [3].

Nucleotide binding domain and leucine-rich repeat containing proteins are members of the Nucleotide oligomerisation domain (NOD)-like receptor (NLR) family out of which NLRP1, NLRP3 and NLRP4 have been characterized as multiprotein complexes acting as "inflammasomes." In general, the NLRs contain three major domains: leucine-rich-repeats, which sense the ligands (LRR), NACHT domain which mediates oligomerization, and an effector domain which is comprised of one of the following three domains: pyrin, caspase activation and recruitment domains (CARD) and baculovirus IAP repeat domain. A complete assembly of the inflammasome is triggered by the oligomerization of NLRs through the NACHT domain upon sensing of microbial or danger signals through the LRRs. For activation of the inflammasome the apoptosis-associated speck-like protein containing a CARD (ASC) is required. ASC recruits the procaspase-1. Procaspase-1cleaves itself and turns into caspase-1. Caspase-1 then cleaves precursors of various pro -inflammatory cytokines such as IL- $1 \beta$ and IL-18. Both IL- $1 \beta$ and IL-18 promote the production of inflammatory mediators including interleukin-6.

\section{The hypothesis}

The proposed hypothesis is that increased activity of inflammasomes as osmosensors is the cause of CE in diabetic ketoacidosis. This means that osmosensing activity of the nucleotide binding domain and leucine-rich repeat containing proteins (NLRP) is the cause of DKA related CE. The osmotic effect of high glucose can activate NLRP's. Activated NLRP's produce interleukin-1 which can via disruption of the cytoskeleton of endothelial cells open intercellular junctions leading to leakage of fluid into the interstitium causing CE. This hypothesis contradicts previous investigators who proposed as theoretical explanations for CE in DKA excessively rapid reduction of extracellular osmolality by intravenous fluids causing cytotoxic cell swelling by osmotic shift of water into cells or cerebral ischemia.

\section{Evaluation of the hypothesis}

\section{Evidence supporting the hypothesis}

- In bone marrow derived murine macrophages immunoblot analysis of cell lysates showed that addition of $\mathrm{NaCl}$, glucose or sorbitol (i.e. $200 \mathrm{mOsm}$ ) into the culture medium induced activation of NLRP's as evident from the cleavage of caspase- 1 into the active subunit p10 and its release into the culture medium. This caspase- 1 cleavage was also demonstrated in the macrophage cell line J774 [4].

- The levels of mature IL-1 $\beta$ and IL-18 were also elevated in

Correspondence to: Michael Eisenhut, Luton and Dunstable University Hospital NHS Foundation Trust, Lewsey Road, Luton, LU4ODZ, United Kingdom, E-mail: michael_eisenhut@yahoo.com

Key words: NLRP, cerebral edema, systemic inflammatory response syndrome

Received: September 18, 2016; Accepted: October 10, 2016; Published: October 13,2016 
culture medium of primary cultures of rat cortical neurons which were treated with high glucose $(50 \mathrm{mM})$. The expression of three essential components of the NLRP1 inflammasome complex, namely, NLRP1, ASC, and caspase 1, was also upregulated in vivo and in vitro under high glucose. Silencing the ASC gene prevented the caspase-1 activation, and inhibiting caspase 1 activity blocked hyperglycemiainduced release of inflammatory factors and neuron injury [5].

- In DKA interleukin-1 levels were found to be elevated with the highest level detectable in peripheral blood within the first 8 hours after admission [6]. Interleukin-1 can interfere with the integrity of the cytoskeleton, which can open intercellular junctions thus leading to extravasation of fluid and interstitial edema [7]. In two cases of fatal CE associated with DKA reported previously features of disruption of the $\mathrm{BBB}$ were detectable and manifested by the absence of tight junction proteins (occludin, claudin-5, ZO-1 and JAM-1) in the parenchymal blood vessels, as well as albumin extravasation in examined brain areas [8]. Another study in thirteen children with DKA showed that whole brain and regional blood-brain barrier permeability increased in the majority during DKA management particularly in the frontal region as measured by serial paired contrast-enhanced perfusion (gadolinium) and diffusion magnetic resonance imaging [9].

- The fact that cerebral oedema can occur in type II diabetes before institution of any treatment [10] with its absence of autoimmunity and keto-acidosis supports the unique role of hyperglycemia in the pathogenesis of cerebral oedema.

\section{Evidence against the hypothesis}

- Interleukin-6 levels were more markedly elevated than interleukin-1 in the first 24 hours and higher in the acute phase of moderately to severe DKA compared to mild DKA as opposed to IL-1, which was higher at baseline in patients with mild DKA [6].

- Initial serum glucose concentrations were not higher in DKA patients with CE compared to random controls without CE [2].

- There is experimental evidence that the beta-hydroxybutyrate (BHB) as released in DKA can inhibit NLRP inflammasomes. It is a potent inhibitor of NLRP3 by preventing potassium efflux and reduction of ASC oligomerization and speck formation. BHB reduced the NLRP3 mediated IL-1 $\beta$ and IL- 18 production in human monocytes and attenuated caspase- 1 activation and IL-1 $\beta$ secretion in mouse models of NLRP3 mediated diseases [11]. This effect should reduce the activity of this inflammasome in DKA.

\section{Evaluation of the hypothesis}

To confirm the role of NLRP's in CE in DKA, cerebral MRI studies in children with DKA could be conducted. Patients NLRP activity and expression could be measured in macrophages isolated from peripheral blood and patients with and without subclinical CE on imaging compared in a prospective controlled study.

Peripheral blood glial fibrillary acidic protein levels (a biochemical marker of brain injury) could be correlated with NLRP activity.

Recently two compounds have been shown to reduce both NLRP3 activity and CE:

Telmisartan is an angiotensin II receptor blocker that shows high affinity for the angiotensin II receptor type 1.In addition to this activity telmisartan acts as a selective activator of peroxisome proliferatoractivated receptor gamma (PPAR- $\gamma$ ) which is linked to inflammasome activation [12].
A single oral dose of 5,10 and $20 \mathrm{mg} / \mathrm{kg}$ telmisartan reduced cerebral edema in the cold induced brain injury model in male C57BL/6 mice and prophylactic administration of telmisartan prevented blood brain barrier impairment, NLRP3, apoptotic speck-containing protein (ASC) and Caspase- 1 activation, as well as IL-1 $\beta$ and IL-18 maturation and improved neurological outcomes [13]. Ruscogenin, a steroid sapogenin derived from Ophiopogon japonicus, decreased edema, improved neurological deficits, reduced evans blue (EB) leakage and upregulated the expression of tight junctions (TJs) in middle cerebral artery occlusion/reperfusion-injured mice. This effect was associated with inhibition of the expression of interleukin-1 $\beta$ (IL-1 $\beta$ ) and caspase-1, and reduction of the expression of NLRP3 [14].

In addition to these two compounds randomized controlled trials could evaluate the effect of interleukin-receptor antagonists on subclinical cerebral edema development as detected on MRI imaging on admission compared to 12 hours after admission.

\section{Discussion}

The biggest study investigating risk factors for CE in DKA compared 61 children with DKA induced CE with 181 randomly selected and 174 age, onset, glucose concentration and initial venous $\mathrm{pH}$ matched controls. Compared to both of these control groups children with CE had lower initial partial pressure of arterial carbon dioxide and higher initial serum urea. The rate of change in the serum glucose concentration during therapy or rates of fluid, sodium or insulin administration were not associated with $\mathrm{CE}$ [2]. Other retrospective studies did equally find no evidence of an association of DKA related CE with treatment $[15,16]$.

Against the fact that treatment is related to CE is the fact that CE in DKA and significant hyperglycemia of type II diabetes can occur before treatment is started [2,10,17-19].

Findings noted so far are rather compatible with an increased duration of DKA being associated with CE. This may explain the association of increased urea levels with CE. Urea in DKA increases with the duration of osmotic diuresis causing progressive dehydration. Prolonged stimulation of upregulation and activity of inflammasomes causing more IL-1 release may be the cause of the link between an increased duration of DKA and an increased risk of CE. The lower carbon dioxide levels found in patients with $\mathrm{CE}$ [2] were found without a lower bicarbonate and are therefore unlikely to be due to more severe metabolic acidosis but are compatible with CE causing hyperventilation rather than hyperventilation causing $\mathrm{CE}$.

The fact that IL-6 levels were more elevated in DKA related CE may simply reflect an earlier significant elevation of IL-1 which triggered the IL- 6 release but was not captured on admission. The alternative hypothesis that cerebral vasospasm is contributing to CE in DKA has support by the fact that IL-1 can cause cerebral vasospasm [20] and cerebral vasospasm has been associated with CE [21].

The postulated effect of the systemic inflammatory response on endothelial and epithelial permeability rather than a cytotoxic effect of osmotic water shifts is supported by the association of DKA with subclinical pulmonary edema [22]. Pulmonary edema pathogenesis in this form of sterile inflammation suggests a cytokine induced opening of capillary endothelial and alveolar epithelial intercellular junctions. The relationship of clinical features to associated radiological and laboratory features is composed in table 1.

The fact that admission glucose level was not higher in patients 
Table 1. Etiological factors in the pathogenesis of cerebral edema in diabetic ketoacidosis.

\begin{tabular}{|l|l|l|}
\hline Clinical features associated with cerebral edema & Laboratory and radiological features & Proposed underlying pathomechanism \\
\hline Dehydration & Elevated urea & $\begin{array}{l}\text { Prolonged action of severe hyperglycemia causing osmotic } \\
\text { diuresis and leading to prolonged activation of inflammasome } \\
\text { mediated interleukin-1 production }\end{array}$ \\
\hline Hyperventilation & $\begin{array}{l}\text { Low partial carbondioxide pressure disproportionate to } \\
\text { metabolic acidosis }\end{array}$ & $\begin{array}{l}\text { Cerebral edema related to effects of longstanding hyperglycemia } \\
\text { inducing inflammasome activation preceeding treatment leads to } \\
\text { hyperventilation }\end{array}$ \\
\hline $\begin{array}{l}\text { Poor memory recall and reduced attention, } \\
\text { neurocognitive changes leading to persistently lower } \\
\text { design recognition }\end{array}$ & $\begin{array}{l}\text { Increased total white matter volume and higher mean diffusivity } \\
\text { in the frontal, temporal and parietal white matter on cerebral } \\
\text { magnetic resonance imaging }\end{array}$ & Inflammasome induced cerebral demyelination \\
\hline
\end{tabular}

with CE [2] may simply reflect the fact that not the absolute glucose concentration and osmotic effects of the glucose or its lowering are related to CE but the duration of the effect of elevated glucose levels on inflammasomes. Prolonged effect of glucose on inflammasomes may also outweigh any inhibitory effect of $\mathrm{BHB}$ on inflammasome function.

In a prospective cohort study comparing 36 DKA and 59 non-DKA patient's cerebral MRI images and neurocognitive state found increased total white matter volume and higher mean diffusivity in the frontal, temporal and parietal white matter in DKA patients. Total white matter volume decreased over the subsequent six months. Changes in brain volume were associated with poor memory recall and reduced attention at 6 months [23]. Even uncomplicated DKA may induce neurocognitive changes leading to persistently lower design recognition [24]. Inflammasome activation can explain white matter changes and associated neurocognitive deterioration: Inflammasome activation has previously been shown to be associated with demyelination [25].

There is therefore a case for double blind, placebo controlled, randomised trials using telmivarsatan in DKA to prevent neurocognitive impairment associated with clinical and subclinical CE. Telmivarsatan has safety record in humans permitting its use for this purpose without further animal experiments in patients with DKA in such trials. Assessment of outcome could include MRI changes, levels of peripheral blood glial fibrillary acidic protein and results of neurocognitive testing.

\section{Conflict of interest}

There was no conflict of interest or funding in the production of this manuscript.

\section{References}

1. Poovazhagi V (2014) Risk factors for mortality in children with diabetic keto acidosis from developing countries. World J Diabetes 5: 932-938. [Crossref]

2. Glaser N, Barnett P, McCaslin I, Nelson D, Trainor J, et al. (2001) Risk factors for cerebral edema in children with diabetic ketoacidosis. The Pediatric Emergency Medicine Collaborative Research Committee of the American Academy of Pediatrics. $N$ Engl J Med 344: 264-269. [Crossref]

3. Glaser NS, Wootton-Gorges SL, Buonocore MH, Marcin JP, Rewers A, et al. (2006) Frequency of sub-clinical cerebral edema in children with diabetic ketoacidosis. Pediatr Diabetes 7: 75-80. [Crossref]

4. Ip WKE, Medzhitov R (2015) Macrophages monitor tissue osmolarity and induce inflammatory response through NLRP3 and NLRC4 inflammasome activation. Nature Communications 6: 6931. [Crossref]

5. Meng XF, Wang XL, Tian XJ, Yang ZH, Chu GP, et al. (2014) Nod-like receptor protein 1 inflammasome mediates neuron injury under high glucose. Mol Neurobiol 49 : 673-684. [Crossref]

6. Karavanaki K, Karanika E, Georga S, Bartzeliotou A, Tsouvalas M, et al. (2011) Cytokine response to diabetic ketoacidosis (DKA) in children with type 1 diabetes (T1DM). Endocr J 58: 1045-1053. [Crossref]

7. Eisenhut M, Wallace H (2011) Ion channels in inflammation. Pflugers Arch 461: 401421. [Crossref]
8. Hoffman WH, Stamatovic SM, Andjelkovic AV (2009) Inflammatory mediators and blood brain barrier disruption in fatal brain edema of diabetic ketoacidosis. Brain Research 1254: 138-148. [Crossref]

9. Vavilala MS, Richards TL, Roberts JS, Harvey Chiu, Catherine Pihoker, et al. (2010) Change in blood- brain barrier permeability during pediatric diabetic ketoacidosis treatment. Pediatric Critical Care Medicine 11: 332-338. [Crossref]

10. Morales AE, Daniels KA (2009) Cerebral edema before onset of therapy in newly diagnosed type 2 diabetes. Pediatr Diabetes 10: 155-157. [Crossref]

11. Youm YH, Nguyen KY, Grant RW, Goldberg EL, Bodogai M, et al. (2015) The ketone metabolite $\hat{I}^{2}$-hydroxybutyrate blocks NLRP3 inflammasome-mediated inflammatory disease. Nat Med 21: 263-269. [Crossref]

12. Lee HJ, Yeon JE, Ko EJ, Yoon EL, Suh SJ, et al. (2015) Peroxisome proliferatoractivated receptor-delta agonist ameliorated inflammasome activation in nonalcoholic fatty liver disease. World J Gastroenterol 21: 12787-12799. [Crossref]

13. Wei X, Hu CC, Zhang YL, Yao SL, Mao WK (2016) Telmisartan reduced cerebral edema by inhibiting NLRP3 inflammasome in mice with cold brain injury. J Huazhong Univ Sci Technolog Med Sci 36: 576-583. [Crossref]

14. Cao G, Jiang N, Hu Y, Zhang Y, Wang G, et al. (2016) Ruscogenin Attenuates Cerebra Ischemia-Induced Blood-Brain Barrier Dysfunction by Suppressing TXNIP/NLRP3 Inflammasome Activation and the MAPK Pathway. Int J Mol Sci 17: 1418. [Crossref]

15. Mel JM, Werther GA (1995) Incidence and outcome of diabetic cerebral oedema in childhood: are there predictors? J Paediatr Child Health 31: 17-20. [Crossref]

16. Rosenbloom AL (1990) Intracerebral crises during treatment of diabetic ketoacidosis. Diabetes Care 13: 22-33. [Crossref]

17. Edge JA, Ford-Adams ME, Dunger DB (1999) Causes of death in children with insulin dependent diabetes 1990-96. Arch Dis Child 81: 318-323. [Crossref]

18. Glasgow AM (1991) Devastating cerebral edema in diabetic ketoacidosis before therapy. Diabetes Care 14: 77-78. [Crossref]

19. Couch RM, Acott PD, Wong GW (1991) Early onset fatal cerebral edema in diabetic ketoacidosis. Diabetes Care 14: 78-79. [Crossref]

20. Eisenhut M (2014) Vasospasm in cerebral inflammation. Int J Inflam 2014: 509707. [Crossref]

21. Ducros A (2012) Reversible cerebral vasoconstriction syndrome. Lancet Neurol 11 906-917. [Crossref]

22. Konstantinov NK, Rohrscheib M, Agaba EI, Dorin RI, Murata GH, et al. (2015) Respiratory failure in diabetic ketoacidosis. World J Diabetes 6: 1009-1023. [Crossref]

23. Cameron FJ, Scratch SE, Nadebaum C, Northam EA, Koves I, et al. (2014) Neurological consequences of diabetic ketoacidosis at initial presentation of type 1 diabetes in a prospective cohort study of children. Diabetes Care 37: 1554-1562. [Crossref]

24. Jessup AB, Grimley MB, Meyer E, Passmore GP, Belger A et al. (2015) Effects of diabetic ketoacidosis on visual and verbal neurocognitive function in young patients presenting with new-onset type 1 Diabetes. J Clin Res Pediatr Endocrinol 7: 203-210. [Crossref]

25. Jha S, Srivastava SY, Brickey WJ, Iocca H, Toews A, et al. (2010) The inflammasome sensor, NLRP3, regulates CNS inflammation and demyelination via caspase-1 and interleukin-18. J Neurosci 30: 15811-15820. [Crossref]

Copyright: (C)2016 Eisenhut M. This is an open-access article distributed under the terms of the Creative Commons Attribution License, which permits unrestricted use, distribution, and reproduction in any medium, provided the original author and source are credited. 